\title{
Systematic Topology Selection Method for Multiple-Input DC-DC Converters
}

\author{
Seung H. Choung*, Sungwoo Bae ${ }^{\dagger}$ and Myungchin Kim**
}

\begin{abstract}
A power system designer may have difficulties in choosing a suitable multiple-input converter topology for a specific target application because each multiple-input converter topology presented in the literature has its own advantages and disadvantages. In this perspective, this paper presents a systematic topology selection method for multiple-input converters with three comparison criteria including cost-saving effect, modularity potential and flexibility. Based on these criteria, this paper proposes a strategic flow chart example for choosing a proper multiple-input converter topology. This flow chart will provide a powerful selection tool to a power system designer when he or she chooses a specific multiple-input converter for a given application.
\end{abstract}

Keywords: Multiple-input dc-dc converter, Cost-saving effect, Modularity potential, Flexibility

\section{Introduction}

Multiple-input dc-dc converters (MICs) have been developed to integrate various energy sources with a single common converter. These MICs may provide a costeffective solution and high availability to a power system designer because they can diversify energy sources with a single common converter [1]. Therefore, the combination of different energy sources by these MICs allows a power system designer to easily use renewable and alternative energy sources such as wind turbine, photovoltaic module and fuel cell. An MIC allows a power system designer to effectively integrate wind turbines and photovoltaic modules with a single common converter when he or she determines the site location and size of a target power system [2]. This possible target power system includes wireless telecommunication sites, health care buildings, data centers and sustainable buildings $[1,3]$.

The main objective of this paper is to provide analytical and useful criteria for choosing a proper MIC topology for a specific target application. Several MIC topologies [1], [2], [4]-[16] have been developed to combine multiple power sources and energy storage elements with a costeffective manner in the literature. However, a power system designer may have difficulties in choosing a suitable MIC topology for a specific target application because each MIC topology presented in the literature has its own advantages and disadvantages. Although some MIC topologies were reviewed in [6] and [10], these studies were only focused on the combining methodology of an MIC topology for connecting its input sources. Specifically, the combining strategies used in [6] and [10]

$\dagger \quad$ Corresponding Author: Dept. of Electrical Engineering, Yeungnam University, Korea (sbae@yu.ac.kr)

* Networks Biz, Samsung Electronics, Korea (sh.choung@samsung.com)

** Agency for Defense Development, Korea (abekim81@gmail.com)

Received: November 19, 2015; Accepted: January 14, 2016

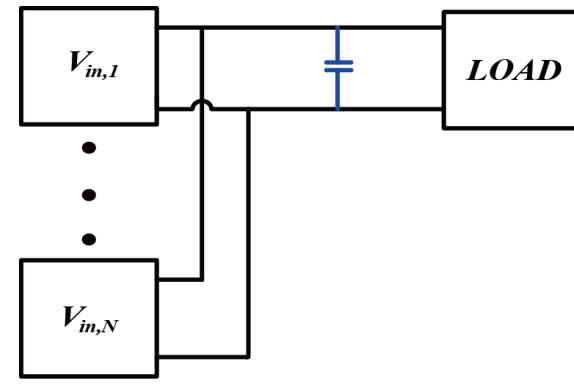

(a) Method I

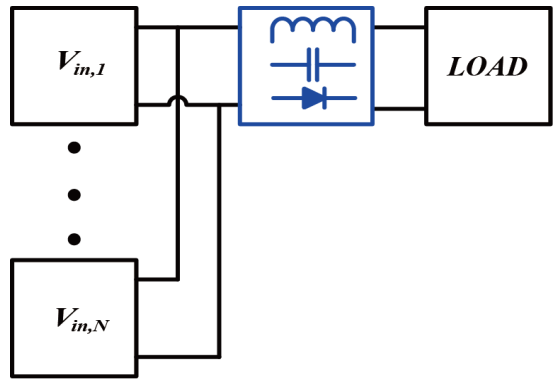

(b) Method II

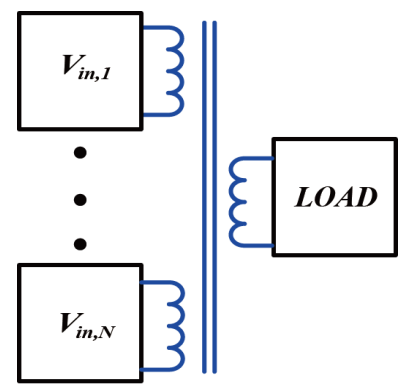

(c) Method III

Fig. 1. Combining methods of an MI converter [4]: (a) sharing an output filter capacitor; (b) sharing switches, inductors and / or capacitors; (c) sharing a magnetic core. 
can be classified by the following three methods: sharing an output filter capacitor [14], sharing some power electronics switches, energy transfer inductor and capacitor $[10,15]$, and sharing a magnetic core $[8,9,11,13]$. Fig. 1 illustrates these three combining methods. This MIC classification methodology with respect to its sharing points and components may be one of essential factors because they largely affect MIC characteristics. However, they are not sufficient to provide definitive MIC topology selection criteria to a power system designer because the same type of MICs with respect to a coupling method may have different characteristics. Therefore, additional comparative aspects when choosing different MICs should be considered in order to realize a reasonable MIC comparison framework.

For comparing MIC topologies, this paper presents three criteria including cost, modularity potential and flexibility. To address these matters, this paper is organized in the following manner: In order to include as many converters as possible in the comparative study, one possible topology is proposed and described in the Section 2. A multipleinput modified Watkins Johnson converter (MIMIWJC) is suggested and briefly described about its circuit operation in the Section 2. The detailed descriptions and reasons for choosing the criteria comparing MICs are described in the Section 3. The results and discussions for the comparative study are explained in the Section 4. Finally, this paper concludes in the Section 5 with the summary and findings.

\section{Multiple-Input Modified Inverse Watkins-Johnson Converter}

This section summarizes a multiple-input modified inverse Watkins-Johnson converter (MIMIWJC) to include it for comparing MICs [4], [16]. Because the input to output voltage conversion ratio of a two-input MIMIWJC is same as that of inverse Watkins-Johnson converter (IWJC) [17] as shown in (1), this converter is called by a multiple-input modified inverse Watkins-Johnson converter (MIMIWJC) as shown in Fig. 2.

$$
V_{\text {out }}=-\frac{D}{1-2 D} V_{\text {in }}, \quad D<0.5
$$

where $V_{\text {in }}$ and $V_{\text {out }}$ represent the input and output voltage of the MIMIWJC respectively, and $D$ denotes its duty ratio.

Fig. 3 shows the voltage conversion ratios of buck-boost converter, boost converter and MIMIWJC. As shown in Fig. 3 , the voltage conversion ratio of an MIMIWJC is higher than that of buck-boost and boost converters. This conversion ratio is helpful in the flexibility comparison category. The detailed description about the flexibility will be discussed in the Section 3.

In the view of a circuit configuration, the biggest

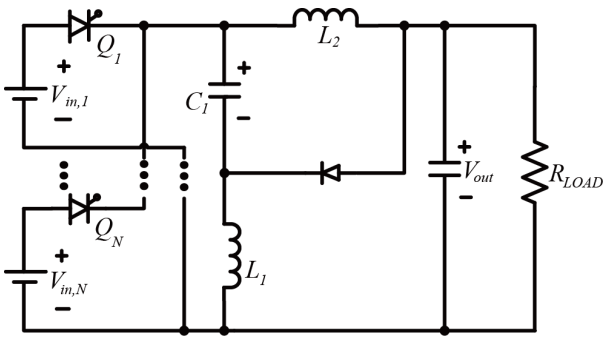

Fig. 2. Circuit topology of an MIMIWJC [4], [16].

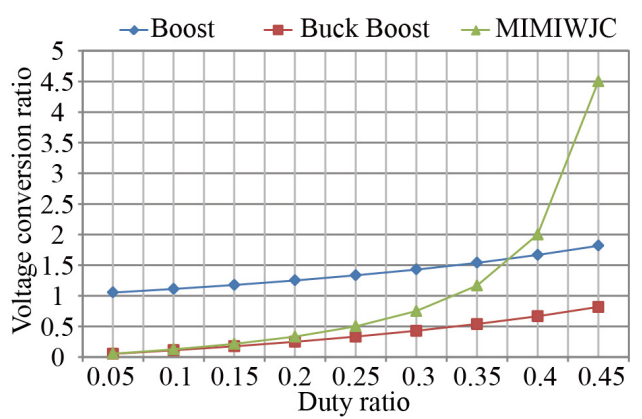

Fig. 3. Voltage gain comparison for three converters.

difference between an unmodified IWJC and a modified IWJC is the existence of a magnetic coupling between two inductors. As shown in Fig. 2, the MIMIWJC does not require a magnetic coupling between two inductors. Instead of breaking the magnetic coupling, a capacitor (i.e., $C_{l}$ ), is inserted into the circuit as shown in Fig. 2 to maintain the same voltage conversion ratio of an IWJC. Actually, a single input modified IWJC schematic diagram can be found in Table 2 as G1(1) in [18]. The elimination of the magnetic coupling in the circuit can provide better modularity although its flexibility might be lower than that with a magnetic coupling. The detailed characteristics of this MIMIWJC will be described in the Section 3 along with other MIC topology candidates.

For the sake of analytical convenience, this section only considered the continuous conduction mode operation and two-input source case of the MIMIWJC. A main switch (i.e., $Q_{x}$ ) in Fig. 2 is a forward-conducting bidirectionalblocking (FCBB) switch such as a gate turn-off thyristor, or a pair of series combination of MOSFET and diode. The possible switching strategy of this main switch is presented in Fig. 4. In Fig. 4, $T$ denotes the switching period, and $q_{x}$ represents the control signals for a switch $Q_{x}$. This switching strategy is called by a time sharing switching method in which more than two input sources cannot deliver power concurrently. The effective duty ratio (i.e., $\left.D_{e f f}\right)$ is defined as the fraction of the switching period when the active switch conducts current. In addition, it is assumed that $V_{i+l}$ is less than $V_{i}$. In other words, the highest voltages source is $V_{l}$ and the lowest one is $V_{N}$. Then, the effective duty ratio becomes as follows:

$$
D_{i_{-} e f f}=D_{i}-D_{i-1} \text {, where } D_{0} \equiv 0 \text {. }
$$




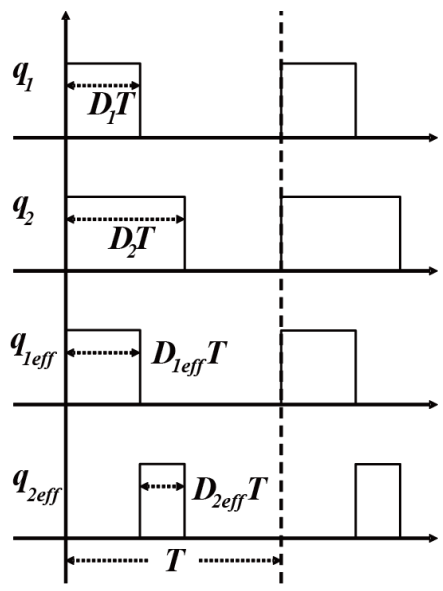

Fig. 4. Two input MIC switching strategy example.

With this switching strategy, the average voltage transfer equation of a MIMIWJC can be easily found by

$$
V_{\text {out }}=-\sum_{i=1}^{N} \frac{D_{i_{-} \text {eff }}}{1-2 D_{N}} V_{\text {in }}, \quad \text { where } D_{N}<0.5
$$

\section{Criteria for a Comparative Study on MICs}

Because the applications of MICs are different from those of single-input converters (SICs), different aspects are required for comparing MICs. Compared with a conventional distributed power system, the most prominent benefit using a MIC is to provide a cost-effective solution with high availability by connecting diversified input sources with modular components. To make the most use of these characteristics, the following three criteria were selected for comparing MICs in this paper: Cost-saving effect, modularity potential and flexibility.

\subsection{Cost-saving effect}

Every MIC has sharing components in its inherent circuit topology while a SIC does not usually have common components in its topology. The largest costsaving effect of an MIC can be achieved by maximizing the number of components in its common stage. Therefore, sharing an output filter capacitor with the input-leg connection of an MIC as shown in Fig. 1(a) tends to provide the least cost-saving effect because the sharing component in the MIC is only the common output filter capacitor [4]. In addition, MIC topologies using a common magnetic core for connecting input sources as shown in Fig. 1(c) can be more expensive than MIC topologies whose common modules consist of switch and passive components as shown in Fig. 1(b) because the production cost of magnetic components is relatively high in a dc-dc converter [4]. Moreover, if the input modules of an MIC are composed of a less number of components, it can be favorable for the production cost perspective when a large number of input power sources are required to be combined.

\subsection{Modularity potential}

The availability of a power system can be defined by (4) where mean uptime represents the mean normal operation time of the system, and mean downtime represents the mean failure time of the system [19]. As seen in (4), the less mean downtime of the system can provide the higher availability of the power system.

$$
\text { Availability }=\frac{\text { Mean Uptime }}{\text { Mean Uptime }+ \text { Mean Downtime }}
$$

In the sense of availability, it is worthwhile to compare which MIC topologies are more generous or easier to make a modular converter than others because the modular converter can reduce its mean downtime. In order to compare modularity potential, one should consider the input combining methods and the complexity of input and common cell configurations of MICs. As regards to a input combining method, sharing an output filter capacitor to interconnect various inputs power source as shown in Fig. 1(a) can be evaluated as the easiest way to make a modular converter. The reason is that not only every input module can be fabricated by the same structure but also output modules can be easily connected with a common capacitor [4]. On the other hand, an interconnection method by a common magnetic core as depicted in Fig. 1(c) is considered to be the most difficult methodology because a specific winding needs to be pre-wired into the common magnetic core when it is required for each input cell [4]. For a circuit structure, a symmetric configuration can increase modularity potential because the same input and output cell configuration can be interchanged.

\subsection{Flexibility}

Different source combinations with an MIC can increase the availability of the target power system. For the higher availability, it is needed to examine that which MIC topology can meet with various input power source requirements. To evaluate flexibility, one should consider input interface types such as current-source interface (CSI) or voltage-source interface [4]. Specifically, an input source such as fuel-cell which requires a low ripple current is better to use a CSI converter. Photovoltaic (PV) modules also require a CSI converter to implement a maximum power tracking control method which uses the PV module current information. In this perspective, a CSI MI converter can have higher flexibility than a VSI MI converter. Also, a topology is better to provide a wide range of the input to output voltage conversion ratio. This is because the wide voltage conversion ratio can offer more 
degree of freedom to a power system designer when choosing the input power sources of a system. In other words, an MIC which has single voltage conversion function such as only step-up or only step-down ability is less flexible than the converter that can have both step upand-down functionalities. An MIC which has a transformer in its inherent topology can be useful in this sense because it can provide a wide-ranged ratio of input-to-output voltage conversion.

\section{Results and Discussions}

The MIC topologies used for the comparative study in this paper were categorized as topology groups described in Table 1. The ten MIC topologies described in Table 1 were selected for the comparative study because they have relatively significant differences both in their circuit topologies and operation modes. For the simplicity of the comparative study, the following points were assumed in this paper: Firstly, the bidirectional characteristics of an MIC were not considered in this topology comparative study because the bidirectional functionality is mostly related with operational techniques. Therefore, for the switches of an MIC, this comparative study did not consider the type of switches but considered the number of switches. Secondly, if there are more than two circuit configurations which are based on the same SIC topology, the circuit that has the smaller total number of components was chosen for the comparative evaluation. Specifically, for a current-source-interface (CSI) filter, the topology without a CSI filter was selected for the comparison because it can increase the production cost although it may increase the flexibility of the circuit. For instance, although an MIb in [15] has a CSI filter, this comparative study used a modified MIb without the CSI filter as shown in Fig. 5. The topologies chosen in this comparative study were presented by the group numbers in Table 1 along with the abbreviated names of topologies and the reference indications.

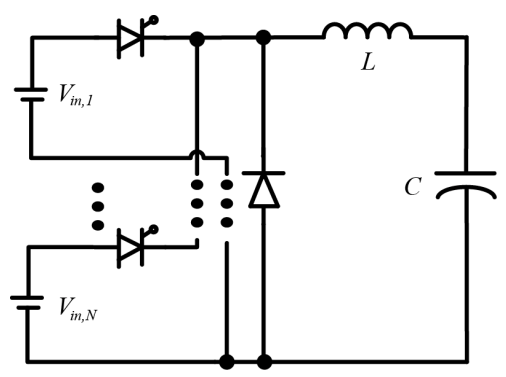

Fig. 5. Circuit diagram of a MIb of which category is a group 1 as described in Table 1.

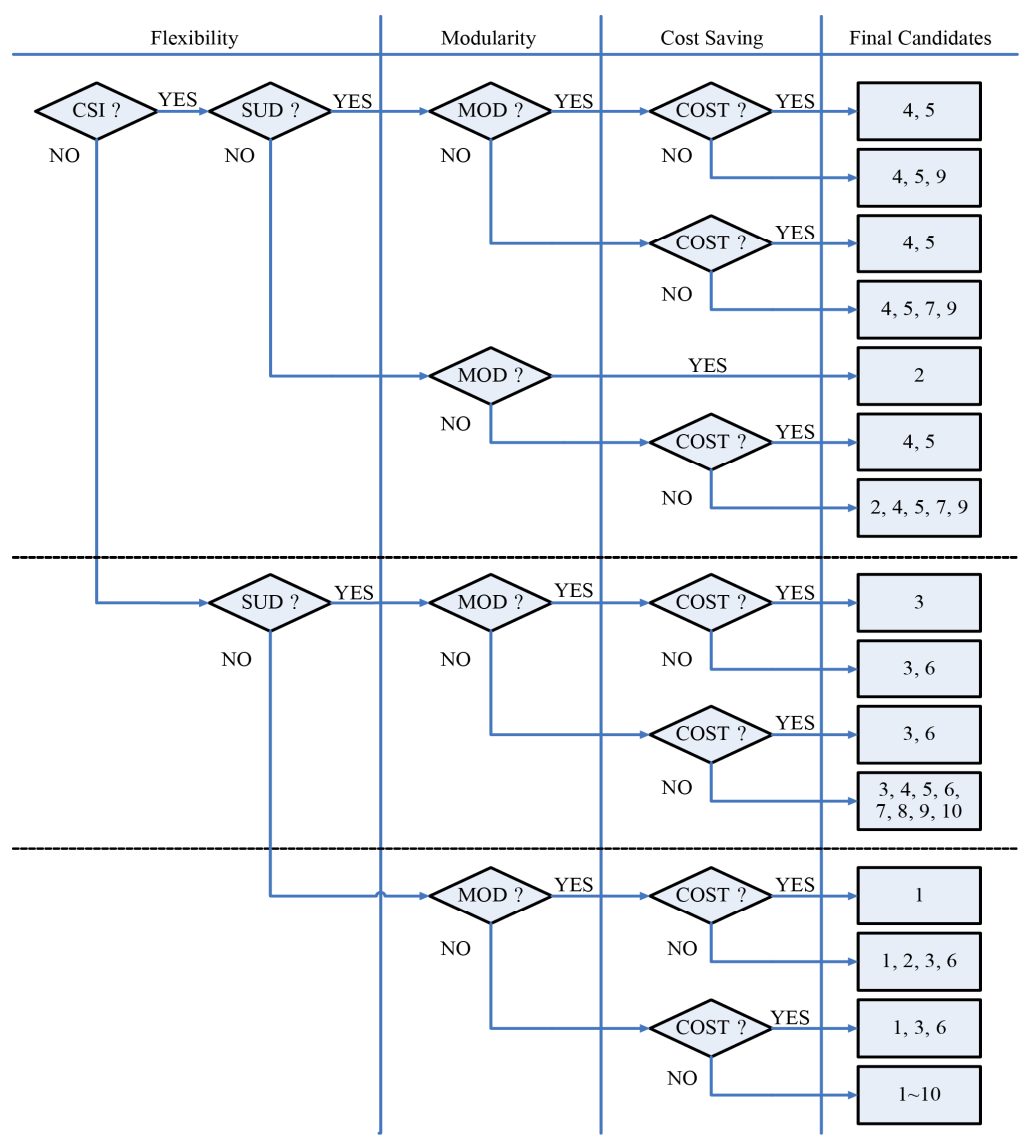

Fig. 6. MIC topology selection flowchart with the emphasis on the flexibility criterion. (A number in the final candidate represents the topology group number described in Table 1.) 
Table 1. List of topology groups for comparing MICs.

\begin{tabular}{c|c|c}
\hline Group & Abbreviation & Description \\
\hline \hline 1 & MIb [15] & MI buck \\
\hline 2 & MIB [2], [14] & MI Boost \\
\hline 3 & MIbB [10], [12] & MI buck-Boost \\
\hline 4 & MIĆUK [4] & MI ĆUK \\
\hline 5 & MISEPIC [4] & MI SEPIC \\
\hline 6 & MIMIWJC [4] & MI Modified IWJC \\
\hline 7 & MIBbB [1] & MI Boost/buck-Boost \\
\hline 8 & MIF [9], [11] & MI Flyback \\
\hline 9 & MICSHB [6]-[8] & MI Current-Source Half Bridge \\
\hline 10 & MIFB [5], [13] & MI Full Bridge \\
\hline
\end{tabular}

Table 2. Supplementary description for Fig. 6.

\begin{tabular}{c|c|c|c}
\hline Criteria & $\begin{array}{c}\text { Condi- } \\
\text { tional }\end{array}$ & $\begin{array}{c}\text { Meaning of Yes in the } \\
\text { Condition }\end{array}$ & $\begin{array}{c}\text { Decision } \\
\text { Method }\end{array}$ \\
\hline \multirow{2}{*}{ Flexibility } & CSI? & $\begin{array}{c}\text { A Current-Source Interface } \\
\text { (CSI) is necessary }\end{array}$ & Elimination \\
\cline { 2 - 4 } & SUD? & $\begin{array}{c}\text { Both Step Up-Down (SUD) } \\
\text { functions are possible }\end{array}$ & Comparison \\
\hline $\begin{array}{c}\text { Modularity } \\
\text { potential }\end{array}$ & MOD? & $\begin{array}{c}\text { It has as higher modularity } \\
\text { (MOD) potential than others }\end{array}$ & Comprison \\
\hline $\begin{array}{c}\text { Cost-saving } \\
\text { effect }\end{array}$ & COST? & $\begin{array}{c}\text { It has more cost-saving effect } \\
\text { than others }\end{array}$ & Comparison \\
\hline
\end{tabular}

Fig. 6 shows a flow chart example of a MIC topology selection method for a specific power system application by the aforementioned criteria including flexibility, modularity potential, and cost-saving effect. As depicted in Fig. 6, the first conditional question in the flow chart is if the target power system requires flexibility. The second conditional step asks if the power system to be designed requires modularity potential. The third conditional inquiry is related with the cost-saving effect of the target power system design. Table 2 describes the meaning of each conditional and decision making process depicted in Fig. 6. The numbers in the final candidate category in Fig. 6 represent the topology groups described in Table 1. Because the flow chart shown in Fig. 6 is designed with emphasizing on flexibility, the first two conditional questions (i.e., CSI? and SUD?) are intended for checking the flexibility requirements of the target system. The CSI? in Fig. 6 means a conditional question if a target power system requires a CSI. The SUD? in Fig. 6 represents a conditional inquiry if the target power system needs both step up-and-down functions. For instance, if the input source of the target power system requires a CSI converter which does not need step up and down abilities, a power system designer may choose 2(MIB), 4 (MIĆUK), 5(MISEPIC), 7(MIBbB) and 9(MICSHB) in Table 1. Among these topology candidates, 2(MIB) can be the topology that has the highest modularity potential. If the target power system requires lower modularity potential than 2(MIB), a power system designer can choose 4 (MIĆUK) or 5(MISEPIC). For another example, if the target power system does not require flexibility, 3(MIbB) can be considered as the topology that has relatively higher modularity potential and cheaper with step up-and- down functions than others. Fig. 6 is just one example of how to use these three criteria for comparing MICs. The conditionals in the flow chart can be changed, or any other conditions can be inserted into the decision process: for instance, the condition related with the existence of transformer for the flexibility category can be inserted into the flow chart. The conditional question about the voltage polarity can also be considered for the flow chart.

\section{Conclusion}

This paper proposed a multiple-input dc-dc converter (MIC) topology selection methodology with three comparison criteria including cost-saving effect, modularity potential and flexibility. These comparison criteria were chosen by considering the benefits and characteristics of an MIC. Based on the proposed criteria, this paper presented a flow chart example which can be used for MIC topology selections. The presented flow chart in this paper compared the ten MIC topologies proposed in the previous literature. The proposed MIC topology selection method with the example flow chart may allow a power system designer to choose a proper MIC topology for a specific target power application with the strategic criteria.

\section{Acknowledgements}

This work was supported by the 2015 Yeungnam University Research Grant.

\section{References}

[1] A. Kwasinski and P. T. Krein, "Multiple-input dc-dc converters to enhance local availability in grids using distributed generation resources," in proc. of APEC 2007 - Twenty Second Annual IEEE Applied Power Electronics Conference, pp. 1657-1663, 2007.

[2] F. Caricchi, F. Crescimbini, A. D. Napoli, O. Honorati, and E. Santini, "Testing of a new DC/DC converter topology for integrated wind-photovoltaic generating systems," in proc. of Fifth European Conference on Power Electronics and Applications, vol. 8, pp. 83-88, 1993.

[3] H. Keiichi, T. Takashi, and M. Seiichi, "Study on Field Demonstration of Multiple Power Quality Levels System in Sendai," in proc. of INTELEC '06. 28th Annual International Telecommunications Energy Conference, pp. 1-6, 2006.

[4] S. H. Choung and A. Kwasinski, "Multiple-input DCDC converter topologies comparison," in proc. of 34th Annual Conference of IEEE Industrial Electronics (IECON 2008), pp. 2359-2364, 2008.

[5] J. L. Duarte, M. Hendrix, and M. G. Simoes, "Three- 
Port Bidirectional Converter for Hybrid Fuel Cell Systems," IEEE Transactions on Power Electronics, vol. 22, pp. 480-487, 2007.

[6] H. Tao, A. Kotsopoulos, J. L. Duarte, and M. A. M. Hendrix, "Family of multiport bidirectional DC-DC converters," IEE Proceedings - Electric Power Applications, vol. 153, pp. 451-458, 2006.

[7] H. Tao, A. Kotsopoulos, J. L. Duarte, and M. A. M. Hendrix, "Multi-input bidirectional DC-DC converter combining DC-link and magnetic-coupling for fuel cell systems," in Fortieth IAS Annual Meeting Conference Record of the 2005 Industry Applications Conference, Vol. 3, pp. 2021-2028, 2005.

[8] G. J. Su and F. Z. Peng, "A low cost, triple-voltage bus DC-DC converter for automotive applications," in proc. of APEC 2005 Twentieth Annual IEEE Applied Power Electronics Conference and Exposition, Vol. 2, pp. 1015-1021, 2005.

[9] K. Kobayashi, H. Matsuo, and Y. Sekine, "Novel Solar-Cell Power Supply System Using a MultipleInput DC/DC Converter," IEEE Transactions on Industrial Electronics, vol. 53, pp. 281-286, 2005.

[10] N. D. Benavides and P. L. Chapman, "Power budgeting of a multiple-input buck-boost converter," IEEE Transactions on Power Electronics, vol. 20, pp. 1303-1309, 2005.

[11] H. Matsuo, L. Wenzhong, F. Kurokawa, T. Shigemizu, and N. Watanabe, "Characteristics of the multipleinput DC-DC converter," IEEE Transactions on Industrial Electronics, vol. 51, pp. 625-631, 2004.

[12] B. G. Dobbs and P. L. Chapman, "A multiple-input DC-DC converter topology," IEEE Power Electronics Letters, vol. 1, pp. 6-9, 2003.

[13] C. Yaow-Ming, L. Yuan-Chuan, and W. Feng-Yu, "Multi-input DC/DC converter based on the multiwinding transformer for renewable energy applications," IEEE Transactions on Industry Applications, vol. 38, pp. 1096-1104, 2002.

[14] A. Di Napoli, F. Crescimbini, L. Solero, F. Caricchi, and F. G. Capponi, "Multiple-input DC-DC power converter for power-flow management in hybrid vehicles," in 37th IAS Annual Meeting. Conference Record of the Industry Applications Conference, vol. 3, pp. 1578-1585, 2002.

[15] F. D. Rodriguez and W. G. Imes, "Analysis and modeling of a two-input DC/DC converter with two controlled variables and four switched networks," in proc. of the 31st Intersociety Energy Conversion Engineering Conference (IECEC 96), vol. 1, pp. 322327, 1996.

[16] S. H. Choung and A. Kwasinski, "Multiple-input modified inverse Watkins-Johnson converter without coupled inductors," in proc. of IEEE Energy Conversion Congress and Exposition (ECCE), pp. 32533260, 2010.

[17] D. A. Grant and Y. Darroman, "Inverse Watkins-
Johnson converter - analysis reveals its merits," Electronics Letters, vol. 39, pp. 1342-1343, 2003.

[18] R. Tymerski and V. Vorperian, "Generation and classification of PWM DC-to-DC converters," IEEE Transactions on Aerospace and Electronic Systems, vol. 24, pp. 743-754, 1988.

[19] Shunji Osaki, Stochastic System Reliability Modeling, World Scientific Publishing, 1985.

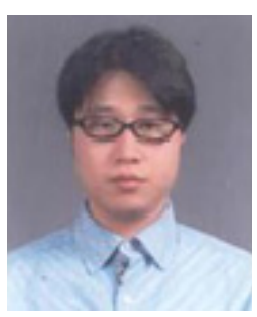

Seung H. Choung received the B.S., M.S. degrees from Kookmin University, Seoul, Korea, in 2000 and 2002 respectively, and Ph.D. degree from the University of Texas at Austin, Texas, in 2011. He joined Networks Biz. of Samsung Electronics, Suwon, Korea, in 2011, where he is a currently senior engineer. His main interest areas are renewable energy, micro grid, multiple-input converter, high efficiency power conversion, and their applications.

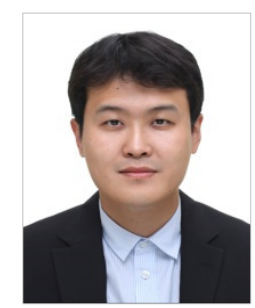

Sungwoo Bae received the B.S. degree from Hanyang University, Seoul, Korea, and the M.S.E. and Ph.D. degrees from the University of Texas at Austin, USA, all in electrical engineering, in 2006, 2009, and 2011, respectively. From 2012 to 2013 , he was a senior research engineer with Power Center at Samsung Advanced Institute of Technology. He has been an Assistant Professor in the department of electrical engineering at Yeungnam University in Korea since 2013. In 2005, Dr. Bae was awarded the Grand Prize at the national electrical engineering design contest by the Minister of Commerce, Industry and Energy of the Republic of Korea.

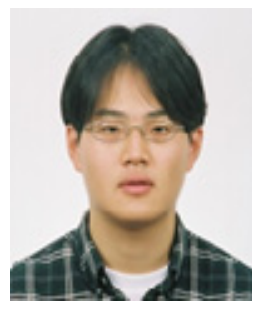

Myungchin Kim received the B.S. and M.S. degrees from Hanyang University, Seoul, Korea and Ph.D. degree from the University of Texas at Austin, USA, all in electrical engineering, in 2004, 2006, and 2015, respectively. Since 2006, he has been with the Agency for Defense Development, Daejeon, Korea, where he is currently a Senior Researcher. His research interests are in avionics, power electronics, on-board power systems, and microgrids. 